\title{
A New Approach to Social Behavior Simulation: The Mask Model
}

\author{
Francesco Osborne \\ Università di Torino, Italy \\ osborne@di.unito.it
}

\begin{abstract}
This paper proposes a new perspective, based on the concept of social masks, for the simulation of a realistic NPC (Non-Player Character) behavior. The Mask Model goal is to support AI techniques for autonomous agents by encouraging or discouraging behaviors according to the social environment and by providing knowledge about possible reactions to the agent actions. In this approach, the NPC tendencies are controlled by the interactions of three overlapping mask layers: selfperception layer, social layer and interpersonal layer. The masks mould the tendencies, the feelings and the ethics of a NPC. By changing the links between characters and masks, a wide variety of different behaviors and story-lines may arise. The paper present an algorithm for the selection of the actions and an example implementation.
\end{abstract}

Keywords: Virtual characters, social behavior, autonomous agents, emergent narrative.

\section{Introduction}

The recent years have seen the outburst of games that reproduce an entire world in which the player can experience daily life, interact with the characters and immerse himself in the illusion of living in a different reality. Therefore the NPCs (Non-Player Characters) have become increasingly important, evolving from mere puppets with some standard answers to more complex entities, able to react to the user's actions.

The approach to the simulation of NPCs social behavior herein presented can be applied as a further enhancement to already existing techniques, and may be particularly useful for supporting autonomous agents. The main issue we want to address is that the NPC behavior is usually weakly correlated to his social and cultural environment and to other character's presence and expected behavior.

For example, let us assume that Alan is brooding hostility toward Bill. In a simple scenario, Alan may tend to assault Bill whenever they meet. However, in the real word, a person must take into account many variables. For example, the two characters may be in a public place, thus Alan cannot attack Bill without compromising himself. Peer pressure and social rules may succeed in convincing him to restrain himself, or to express his hostility only through words (maybe insults) or even hide it completely. If moreover character Cindy, the lady both 
of them are in love with, is present, both men may want to pretend to behave as righteous gentlemen.

Psychology studies have shown that humans tend to exhibit a social performance that leads them to play different roles under different situations (see 445[16]). In the Mask Model the character tendencies are controlled by the interactions of three overlapping mask layers representing the social and the interpersonal interactions influencing a character. A mask can mould the character behavior and its psychological characteristics, it also may contain some "social expectations" that a NPC can use to predict other character's reactions. This knowledge is useful to autonomous agents to build more farsighted and realistic plans.

The paper is organized as follows. Section 2 summarizes the relevant related work, Section 3 introduces the concept of mask, Section 4 shows how mask layers may be used to built a character, Section 5 discusses how the masks affect the selection of an action, Section 6 presents an example of an implemented scenario, Section 7 concludes the paper, pointing out advantages, limits and eventual developments of this approach.

\section{Related Work}

Many works have shown the usefulness of both weakly and strongly autonomous agents in virtual storytelling. The former ones are usually getting directions from a drama manager to pursue the author goals [10 and may use different AI techniques such as case-based reasoning [15, planning [17] or reinforcement learning [12. The strongly autonomous agents [1] try instead to create an emergent narrative thanks to the interactions between themselves, the player and the environment. One of the classic solutions for building autonomous agents is the BDI (Belief-Desire-Intention) architecture, since it enable the agent to "react to an environmental change in many different ways, which do not have to be explicitly specified by designers" 3. A good examples of a storytelling system based on BDI agents is MIST 13 .

Social behavior management is an intriguing challenge for interactive storytelling. Prom Week [1] addresses the problem of creating games based on relationships and social interactions among characters. Similar solutions are found in PsychSim [8, a multi-agent capable system that deals with social interactions based on models of social influence. In Mascarenhas et al. 9] it is noted that "cultural aspects have been largely neglected so far, even though they are a crucial aspect of human societies" and is proposed to use rituals in synthetic characters to generate cultural specific behaviors. Lee and Nass [7] shows that users prefer to interact with computer agents that share the same cultural background. The Storytron technology [2] develops in detail many concepts exposed in this paper, but, unlike in the Mask Model, uses inclination equations for dealing with the character behavior.

Several studies have been conducted on the psychological and social concepts of mask and on social relations as a performance. The concept of mask has been 
the nucleus of the work of the Nobel laureate writer Pirandello [14], who argues that every man is forced to wear a mask by the society and that everyone tends to show a different mask to different people. The concept of social relations as a performance was extensively studied by Goffman [5], who introduced a new outlook on social interactions described through the metaphor of a drama with actors, roles, props, setting, audience and a stage. A good essay on the concepts of mask and dramaturgical social psychology is found in Edgley and Turner [4]. In the work of Strauss [16] we find an in-depth study of this subject and a good description of the social masks according to which "everyone present himself to the other and to himself, and sees himself in the mirrors of their judgment. The masks he then and thereafter presents to the word and its citizens are fashioned upon his anticipation of their judgment."

\section{The Mask}

The Mask Model aims at supporting AI techniques for autonomous agents by encouraging or discouraging behaviors according to the social environment and providing knowledge about possible reactions to the agent actions. Therefore the Mask Model is designed to work with different types of autonomous agents and IA techniques. The basic requirement is that agents must be able to perceive the world around them and execute a set of actions.

The Mask Model can be useful to manage both round and flat characters. Round characters are characters whose personality is fully delineated by the author, while flat characters are only useful in carrying out some narrative purpose or, as in many video games, in offer services and information. We can say that the round characters make the story, but the flat characters make the world come alive.

Using the Mask Model instead of scripts to create and handle a round character permits a more structural approach since it allows to decompose the personality of a character in various components and in different layers (as we will see in Section 4). It also allows the character to reason on the basis of the other characters "social expectations", hence casting him or her in the social and cultural conventions of the world. The Mask Model should bring additional benefits with regard to the management of a multitude of flat characters for which a script approach might be too heavy or too monotonous. We will see how, by combining together a relative small number of simple masks, it is possible to create the illusion of deep behavior, making flat characters more alive and realistic.

According to the presented approach, a mask is defined as a set of characteristics and behaviors expected by a reference entity. Breaking the expected behavior is considered morally wrong by the entity and may lead to penalties. On the contrary, respecting it is encouraged and may bring advantages. Masks can be used to describe relationships, as well as many cultural aspects, like the four types of culture manifestations described by Hofstede []: values, rituals, heroes and symbols. 
Any character can be linked to one or more masks. The link that connects a person to a mask is associated with two values representing the character role and the level of influence that the mask exerts on the character.

The role is needed to discriminate among the behavior expected from different kinds of characters. For example, the mask "culture of kingdom X" may have a different expected behavior for slaves, free men or nobles. In the same way, a young lady will expect a different behavior from her lover or from her acquaintances. Usually the role is a label such as "believer", "noble","servant", "lover", "client".

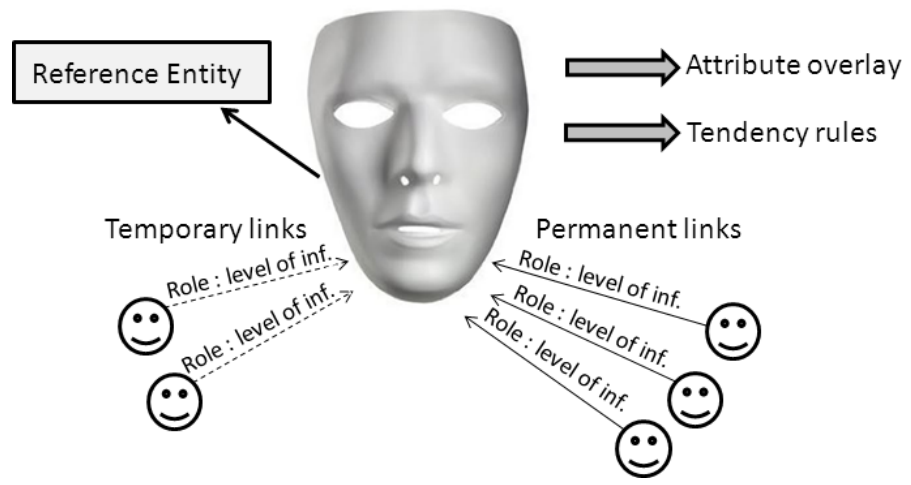

Fig. 1. The mask structure

A character may have more than one role for the same mask and the role can contain a reference to an entity that we call role entity. The latter is obtained by writing the role in the form "role $\rightarrow$ entityID". For example, we can say that Bill's role is not "generic servant" but specifically "servant of Rupert", by using as role "servant $\rightarrow$ Rupert". Thus some suggested reactions to Bill's actions could involve Rupert.

The level of influence is represented by a value between 0 and 1 , according to how much the mask affects the morality, the ethics and the feelings of a NPC. If the reference entity is a religion, a low value would describe a moderate believer, a high value would point to a fanatic ready to even kill for it.

Each character has some persistent masks and may be subjected to the influence of other masks activated by the environment. The persistent masks represent the values, the education and the imprinting that affect a character. They may however be lost or acquired as a result of certain events.

The masks triggered by the environment are activated when the character falls within the sphere of influence of a reference entity, establishing a temporary link. For example, let us imagine a town where people follow strict religious rules and misconduct is regarded as scandalous or even heretical: a character that ordinarily does not follow that religion may decide to adapt his behavior when he enters the town, either for peer pressure or for fear of punishment. 
The masks should be created by abstracting the main philosophies, religions, socially encouraged behaviors linked with different cultures, political views and opinion leaders in the world. A mask should be built when it is presumed that it will have an appreciable number of links or when it is needed for some special reason.

Each mask is composed by three parts (see Figure 1):

- a link to the reference entity,

- an overlay (even partial) on the attributes of the character,

- a set of tendency rules.

The reference entity can be a social entity (such as a government, a religion or a group of people with a common ideology), a single person or even the character himself. We will see a possible classification in the next section.

The overlay allows to change some characteristics of the characters in either an absolute or relative way, for example by setting the attribute "generosity" or "hostility" to a new value, or incrementing or decrementing them by n points. This is particularly useful in implementations that take into account various psychological components associated with an intensity (like "Prom Week" [1]). A mask can have different overlays for different roles and the overlay effect is to be weighted with the level of influence.

A tendency rule dictates an action that is encouraged or prohibited by the reference entity. It is expressed in the form:

rule_id - affected roles $:\{$ precondition $\rightarrow$ action(entity) $\}:$ strength

Its meaning is simple: if the character fills one of those roles when the preconditions are triggered, an action on a certain entity is either suggested or discouraged. An action can have one or more arguments and may also be a mental act used to modify a mental state. The arguments can be entities introduced in the precondition, the role entity, the mask reference entity or the character itself.

Each tendency rule is associated with the rule strength which reflects the significance of the rule for the reference entity and is represented by a value between -1 and 1. A negative value implies that the behavior is discouraged, whereas a positive value spells encouragement.

This mechanism is made flexible by the possibility of using the body of a rule as a precondition to another rule, thus allowing to model the expected social reactions to an action. For example, we can have rule_1 - citizen : $\{$ true $\rightarrow$ steal(object) $\}:-0.5$, that formalizes that stealing is always morally wrong for this mask. Then we can add rule_2 - policeman : $\left\{\left(\right.\right.$ believe $\left(\right.$ rule $\left._{-} 1(X)\right) \rightarrow$ $\operatorname{arrest}(X)\}: 0.8$, to dictate that a cop will have the strong duty of arresting the thieves. Another possibility is to set a precondition about any action with a strength less or greater than a fixed value. For example any action by a certain role that has a strength $<-0.5$ will be considered very immoral and thus cause a standard negative response.

It is also important to notice that a character has usually access to the rules denoting the other roles, for example a thief will know that a cop is supposed to arrest him. This knowledge can be effectively used for a wiser selection among plans that involve other people, as it will be shown in Section 5 . 


\section{The Mask Layers}

An important feature of this approach is that the character tendencies are controlled by three overlapping mask layers (see the example in Figure 2):

- self-perception layer,

- social layer,

- inter-personal layer.

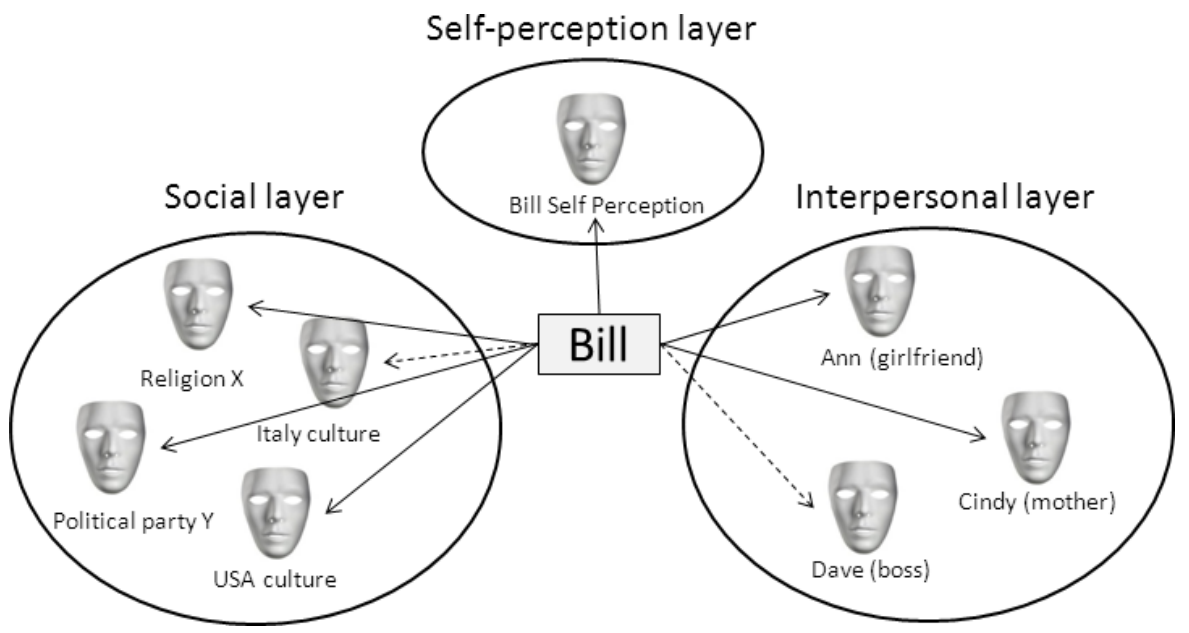

Fig. 2. A example of mask layers. Solid arrows represent the permanent links, dashed arrows temporary links. From the latter we can see that Bill is in the sphere of influence of Italy and his boss. Role and influence were omitted to simplify the picture.

The self-perception layer consists of a single mask that represents the idea that a character has about himself. It is useful to modulate the deepest trends of a character and is also needed to model elusive concepts as self-deception. In a real implementation it is neither needed nor advisable to create a mask for each character, but it is suggested to build a mask at least for a handful of major characters or opinion leaders of the simulated world. For the minor characters it is much easier to use a neutral mask and let their behavior be controlled by other masks.

The social layer is made up of all the masks related to the macro-social aspects. The masks of this level represent both the values of a person and his respect for the rules of the social environment. In the first case, the masks are always active, or at least as long as such values are alive: it is the case of the faith in a religion or the loyalty to a certain group. In the second case, the masks will be activated only when the character enters the sphere of influence of the reference entity. 
The inter-personal layer collects all those masks that have as reference entity another character. As for the social layer, they may be always active or get activated only in the presence of this particular character. The first case models the behavior of a person so strongly influenced by a second character that he acts according to that persons values and expectations. For example, a widower may continue to act as his wife wanted him to do, as a tribute to her memory. Or a soldier could be so influenced by his commander to pattern his behavior after him. In the second case a mask is activated by the presence of a person and the mechanism is the same as the one described for the environment.

\section{Solving Conflicts and Selecting an Action}

In many multi-agent systems, an agent selects a goal to pursue and consequently applies the plans necessary to achieve it. However, this does not sit well with the human way of thinking. Often the actions needed to achieve a goal are as important as the goal itself for a human being. So the focus must be shifted from the simple goal to more complex "goal-plan" pairs.

In the BDI architecture it is common to have different plans that can accomplish a goal and the criterion for choosing them is usually left to individual implementations. For example, if the goal is "obtain 100 gold coins" we can do that by finding a job, by stealing, by asking a friend for them. Any of the plans may have different sub-goals and sub-plans from which to choose. In our approach the agent should look at the different paths leading to a goal to find and execute the one which has the greatest likelihood for it in a certain social context. Of course, the very same technique can be used also for basic agents who select only atomic actions.

This requires the evaluation of both rational and emotional/ethical aspects. In fact a mask can either recommend or discourage a certain action regardless of the goal. This can lead to irrational behaviors: this kind of NPC can perform acts against his own interest and purposes, for sentimental, ideological or religious reasons. In addition, different masks could be in contradiction with each other: for example, love may encourage actions in conflict with religion or political ideology.

The likelihood of an action is calculated by combining the weight of the pros and cons and the weight of the perceived moral soundness of the action.

The weight of pros and cons is obtained by estimating the total gain achieved by the character because of an action. By gain we mean any improvement to the character condition. This can be attained in many ways, for example by reaching a previously established goal, or by improving his or her health or by increasing the level of intimacy with another character. These considerations must be translated into a value between -1 and 1 . The estimate gain of an action will be negative if such an action has a probability to damage the character. However we should take into consideration two types of gain, a direct one and an ensuing one. The first include the immediate benefits or harms obtained through an action. The second are the benefits or damages resulting from the 
actions that can be set in motion by the tendency rules which suggest a reaction to the original behavior.

To compute the ensuing gain we need to introduce the concept of visibility, probability and estimation of the success of an action. Any action performed in the world may be visible to some entity while remaining concealed to others. Therefore, before selecting an action, the character needs to estimate its possible spectrum of visibility. This value can be expressed as a percentage that reflects the probability for a certain action to be discovered by an involved role. It is worth noting that the estimation of visibility can be made only on the basis of what the character actually knows. In fact the character may think that his or her actions have no visibility whereas it is not so. The other factors to be taken into consideration are the probability of the ensuing actions and their estimation of success: for example, a thief should estimate the probability that a policeman may decide to arrest him after learning of the violation and the probability that he will be successful in doing this.

Combining this factors we obtain a value that we call ego esteem E:

$$
\mathrm{E}=\alpha \mathrm{g}+(1-\alpha) \sum_{i=1}^{n} \mathrm{v}_{i} \mathrm{p}_{i} \mathrm{~s}_{i} \mathrm{~g}_{i}
$$

where $\mathrm{g}$ is the direct gain; $\mathrm{v}_{i}, \mathrm{p}_{i}, \mathrm{~s}_{i}$ are respectively the visibility of the initial action, the probability and the estimation of success of the ensuing action; $\mathrm{g}_{i}$ is the ensuing gain for the $\mathrm{i}$-th rule (with i ranging from zero to the maximum number $n$ of rules activated by the initial action). In the absence of other pieces of information, the probability of the ensuing action can be derived from the strength of the tendency rule. The parameter $\alpha$ is a measure of the character impulsivity and varies from 0 to 1 . A character with a high $\alpha$ will dive into an action without thinking too hard about the consequences, whereas a character with a low $\alpha$ will consider carefully all its possible outcomes.

The weight of the perceived moral soundness of the action depends both on the influence of the relevant masks and on the strength of the tendency rules associated with the action for each mask. The summation of the products of these two values associated with every involved rule gives the weight of the perceived moral soundness of an action, which we call super-ego esteem S:

$$
\mathrm{S}=\sum_{i=1}^{n} \mathrm{r}_{i} \mathrm{~m}_{i}
$$

where $r_{i}$ is the strength of the $\mathrm{i}$-th rule (with i ranging from zero to the maximum number $n$ of rules activated by the initial action) and $m_{i}$ is the influence of the relative mask.

If a character is strongly influenced by a mask, even a relatively low strength rule will be followed. Conversely, if a character is only slightly affected by a mask, he or she will tend to follow only the most important rules associated with it, or even disregard them at all. Therefore $S$ will be positive if the action is encouraged and negative if it is discouraged. 
In some cases the masks may end up in conflict with each other. For example a cop may fall in love with Bonnie, a thief. The mask which has as reference entity "the culture of city X" will push him to arrest all those who steal, whereas the mask relative to the reference entity "Bonnie" will refrain him from taking that very same action against his loved one.

Finally the likelihood of an action $\mathrm{L}$ is estimated by combining $\mathrm{E}$ and $\mathrm{S}$.

$$
\mathrm{L}=\beta \mathrm{E}+\gamma \mathrm{S}
$$

where $\beta$ and $\gamma$, varying between 0 and 1 , represent respectively the character rationality (in a selfish sense) and morality. A character with a high $\beta$ and a low $\gamma$ will tend to act in a rational way to pursue a personal advantage, even disregarding his moral and his ideals. A character with a high $\gamma$ and a low $\beta$ will be idealistic and will be ready to behave irrationally and even to sacrifice himself for his values or his emotions.

The resulting $L$ value is used to trigger the selection between concurrent actions. The mechanism for the selection of a "goal-plan" pair based on the $L$ value computed for each action may change according to the agent's architecture and implementation. However we suggest to select the "goal-plan" pair for which the sum of $\mathrm{L}$ values is maximum.

\section{Example of Implemented Scenario}

The Mask Model has been implemented in a simple text-based prototype in java. As an example in the following we will show how a simple scenario can have many outcomes by changing only the environment and the mask links.

Let us consider three character: Ann, Bill and Cedric. Ann, while being engaged with Bill, has also an affair with Cedric. We created three masks: "Athens culture", "Sparta culture" and a generic "Romantic Relationship" mask.

The first two masks describe some social rules of a very pacific culture and those of a more aggressive one. The former contains rules that in case of hostility suggest to talk and discourage to fight. The latter has an opposite philosophy. They both have the special role "is_here" that denotes the temporary link that is created when a character enter in their sphere of influence. The romantic relationship mask (from now on $\mathrm{RR}$ ) encourages actions like giving a present or a kiss and discourage actions like betraying or harming the other person. Furthermore as self-perception mask we used a neutral mask for every character, in order to see more clearly the effect of the other masks.

With such a minimal initial set we already have 1331 possible link configurations (assuming that no one will have a cultural background including both Athens and Sparta) and two possible spheres of influence on our environment: thus 2662 possibilities that may, at least theoretically, lead to different storylines.

The initial situation is that Bill discover Ann and Cedric kissing, and from this seed many different things can follow. We ran different simulations by changing the links and the environment. 


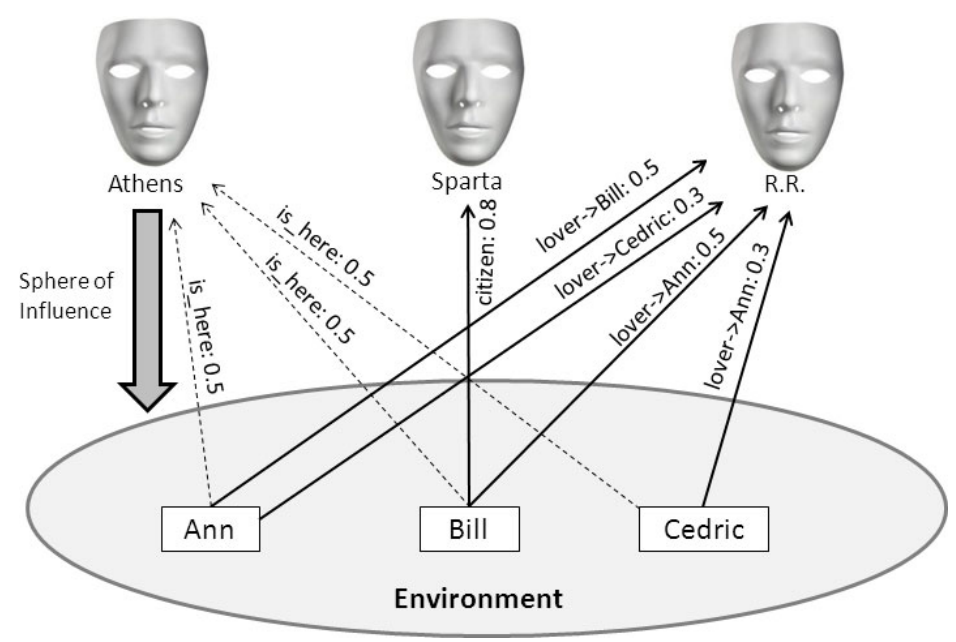

Fig. 3. A possible configuration of the example scenario. Solid arrows represent the normal links, dashed arrows temporary links. Here the environment is subject to "Athens culture" sphere of influence.

The input data are the environment and a set of links that dictate the tendency and attitude of the characters like: Environment: Sparta; Bill is lover $\rightarrow$ Ann $: 0.5$ $\Rightarrow$ R.R. ; Cedric is lover $\rightarrow$ Ann : 0.3 $\Rightarrow$ R.R. ; Ann is lover $\rightarrow$ Bill : $0.5 \Rightarrow$ R.R.; Ann is lover $\rightarrow$ Cedric : $0.3 \Rightarrow R . R$. For the sake of the example we made every role and mask "public", that is every character knows the other character masks. In a realistic scenario a character have to infer this information, for example, from the other characters outlook or behavior.

The output is a sequence of actions like:

"Ann and Cedric are kissing, Bill sees that Ann and Cedric are kissing, Bill is hostile to Ann, Bill is hostile to Cedric, Bill attacks Cedric, Cedric dies, Bill tells Ann 'Go away', Ann cries."

In this situation the environment lies in the sphere of influence of the Sparta culture, thus Bill has no problem in physically attacking Cedric, but at the same time the mask that represents his love for Ann prevents him to hurt her. If we just change the environment to Athens the outcome will be different:

"[first steps are the same], Bill is hostile to Cedric, Cedric tells Bill 'I am sorry', Bill tells Cedric 'Go away', Cedric goes away, Bill tells Ann 'Go away', Ann tells Bill 'I am sorry'."

In the previous cases Ann, Bill and Cedric had no cultural background themselves, therefore they adapt their behavior to the environment. But what will happen if we add "Bill is citizen : $0.8 \Rightarrow$ Sparta", making Bill a devout spartan? This is the situation show by Figure 3 .

"[first steps are the same], Bill is hostile to Cedric, Cedric escape, Bill tells Ann 'Go away', Ann tells Bill 'I am sorry'." We can see that here Cedric does not even try to argue, but he escapes immediately. The reason is the "social 
expectation" reasoning: Cedric knows that Bill is a spartan and knows that spartans do not hesitate to take revenge for a betrayal in a violent way and since he is under the Athen influence he decides to avoid the battle.

We can also change some quantitative value, like reinforcing the link from Ann to the R.R. mask for the "lover $\rightarrow$ Bill" role bringing it up to 1 , thus making Ann desperately in love with Bill. Furthermore we hide roles and masks to prevent Cedric to predict the actions of the rival.

"[first steps are the same], Bill is hostile to Cedric, Cedric tells Bill 'I am sorry', Bill attacks Cedric, Ann defends Cedric, Ann dies, Cedric cries, Bill cries, Cedric is furious with Bill, Cedric attacks Bill, Cedric dies."

We can thus see how simply changing the link and their strength encourages every time a different behavior, yielding a lot of different stories and situations.

\section{Conclusion}

In the previous sections we presented the Mask Model and discussed how it aims at simulating complex social behaviors. The combinatory explosion, the nightmare of many storytelling systems, may here be used as an ally, to build dramatic situations and articulate behaviors by combining in different way masks, roles and influence values. If the domain masks are well built, the behaviors will turn out deep and realistic.

In my opinion, the strong points of this method may be found in:

- a wide variety of simulated behaviors,

- the "social expectations" included in the masks can be used by a character to create more realistic plans,

- the ability to easily create flat characters by combining masks together.

The third goal is achieved by defining a set of typical masks to generate a large number of stereotyped characters. For example, it is possible to generate a crowd of believers in religion $\mathrm{X}$, or a sect that follows the political ideal $\mathrm{Y}$. It may be useful to introduce some small random differences between these characters, but, thanks to the masks, the unity of purpose will be maintained. In the same way a combination of masks can be used to generate characters with certain characteristics.

The main limit of this approach is that it needs a preparatory work on the domain to create the masks and set up the initial links. This work may be not trivial, thus it is necessary to weigh carefully costs and benefits. Especially for very limited story world the Mask Model may be a overkill.

As far as future developments, we are working on finding the best strategy to generate and maintain the links between character and masks and on being able to foresee, at least partially, their impact on the story world. The final cost of the Mask Model will depend on how efficiently it will be possible to do this.

We plan to implement a system which aims at creating interactive stories by exploiting the Mask Model. The NPC will be allotted some degrees of freedom and the author's goals will be satisfied by a drama manager acting on the masks, the links and the environment. 


\section{References}

1. Aylett, R.S., Louchart, S., Dias, J., Paiva, A., Vala, M.: FearNot! - An Experiment in Emergent Narrative. In: Panayiotopoulos, T., Gratch, J., Aylett, R.S., Ballin, D., Olivier, P., Rist, T. (eds.) IVA 2005. LNCS (LNAI), vol. 3661, pp. 305-316. Springer, Heidelberg (2005)

2. Crawford, C.: Chris Crawford on Interactive Storytelling. New Riders, Berkeley (2004)

3. Davies, N., Mehdi, Q., Gough, N.: Creating and visualising an intelligent npc using game engines and ai tools. In: Proc. 19th European Conference on Modelling and Simulation, Riga, Latvia, pp. 721-726 (2005)

4. Edgley, C., Turner, R.: Masks and social relations: An essay on the sources and assumptions of dramaturgical social psychology. Humboldt Journal of Social Relations 3, 4-12 (1975)

5. Goffman, E.: The Presentation of Self in Everyday Life. Doubleday Anchor Books, Garden City (1959)

6. Hofstede, G.H.: Culture's consequences: Comparing values, behaviors, institutions, and organizations across nations, 2nd and enlarged edition. Sage, Thousand Oaks (2001)

7. Lee, E.J., Nass, C.: Does the ethnicity of a computer agent matter? An experimental comparison of human-computer interaction and computer-mediated communication. In: Proceedings of the 1st Workshop of Embodied Conversational Characters, WECC 1998 (October 1998)

8. Marsella, S., Pynadath, D., Read, S.: Psychsim: Agent-based modeling of social interactions and influence. In: Proc. of the International Conference on Cognitive Modeling, pp. 243-248 (2004)

9. Mascarenhas, S., Dias, J., Afonso, N., Enz, S., Paiva, A.: Using rituals to express cultural differences in synthetic characters. In: Sierra, C., Castelfranchi, C., Decker, K.S., Sichman, J.S. (eds.) AAMAS (1), pp. 305-312. IFAAMAS (2009)

10. Mateas, M., Stern, A.: A behavior language for story-based believable agents. IEEE Intelligent Systems 17, 39-47 (2002)

11. McCoy, J., Treanor, M., Samuel, B., Tearse, B., Mateas, M., Wardrip-Fruin, N.: Authoring game-based interactive narrative using social games and comme il faut. In: Proc. of the 4th International Conference and Festival of the Electronic Literature Organization: Archive and Innovate (ELO 2010), Providence, Rhode Island, USA (2010)

12. Nelson, M.J., Roberts, D.L., Isbell Jr., C.L., Mateas, M.: Reinforcement learning for declarative optimization-based drama management. In: Proceedings of the Fifth International Joint Conference on Autonomous Agents and Multiagent Systems, AAMAS 2006, pp. 775-782. ACM, New York (2006)

13. Paul, R., Charles, D., McNeill, M., McSherry, D.: Mist: An interactive storytelling system with variable character behavior. In: Aylett, R.S., Lim, M.Y., Louchart, S., Petta, P., Riedl, M. (eds.) ICIDS 2010. LNCS, vol. 6432, pp. 4-15. Springer, Heidelberg (2010)

14. Pirandello, L.: Maschere Nude. Mondadori, Milano (1986)

15. Sharma, M., Ontañón, S., Mehta, M., Ram, A.: Drama management and player modeling for interactive fiction games. Computational Intelligence 26(2), 183-211 (2010)

16. Strauss, A.: Mirrors and masks: the search for identity. Transaction Publishers, New York (1997)

17. Young, R.M., Riedl, M.O., Branly, M., Jhala, A.: An architecture for integrating plan-based behavior generation with interactive game environments. Journal of Game Development 1(1), 1-29 (2004) 